\title{
SECTOR COLOMBIANO DE CUERO, CALZADO Y MARROQUINERİA: UN ANÁLISIS DE LA DINÁMICA COMERCIAL Y LAS VENTAJAS COMPARATIVAS RESPECTO A VENEZUELA (1995-2017)*
}

\section{Colombian Leather, Footwear and Leather Goods Sector: an analysis of commercial dynamics and comparative advantages over Venezuela (1995-2017)}

Rémi Stellian**, William Guillermo Jiménez ${ }^{* * *}$, Pedro Nel Páez****

Recepción: 3 de octubre de 2018. Aceptación: 23 de febrero de 2019.

DOI: http://dx.doi.org/10.21017/Rev.Repub.2019.v27.a71

\section{RESUMEN}

El presente trabajo analiza cómo han evolucionado los intercambios del sector colombiano de Cuero, Calzado y Marroquinería (CCM) con Venezuela, y en qué medida dicha evolución puede explicarse por las ventajas comparativas del sector. Se evidencia que el sector CCM pudo seguir registrando un excedente comercial frente a Venezuela, a pesar de la crisis económica y política en este país. El calzado y el cuero son las dos categorías de productos que impulsaron de manera decisiva este excedente. Esto se confirma por el cálculo de las ventajas comparativas del sector CCM, a partir de un indicador de ventajas comparativas

\footnotetext{
* El presente trabajo es producto de investigación de los grupos Riesgos Financieros y Métodos de Valoración de Empresas-RISVAL, Pontificia Universidad Javeriana; Reflexión Económica, Administrativa y Contable (REAC) de la Fundación Universitaria los Libertadores, y el grupo Política, Derecho y Gestión Pública, línea Competitividad, Desarrollo Económico y Productividad, de la Escuela Superior de Administración Pública (ESAP).

** Doctor en Ciencias Económicas y Sociales, Universidad de Friburgo (Suiza) profesor, Departamento de Administración de Empresas, Pontificia Universidad Javeriana (Colombia); integrante del Grupo de Investigación Riesgos Financieros y Métodos de Valoración de Empresas-RISVAL. Correo electrónico: rstellian@javeriana.edu.co

*** Pos-Ph. D. en Derecho de la Vrije Universiteit Amsterdam y Ph. D. en Ciencias Políticas de la Universidad de Santiago de Compostela; profesor titular de la Escuela Superior de Administración Pública -ESAPy de la Universidad Libre. Correo electrónico: willjime@esap.edu.co, gjimen00@mail.com

**** Pos-Ph. D. en Economía de la Vrije Universiteit Amsterdam y Ph. D. en Economía de la Universidad Nacional de Colombia;profesor asociado de la Fundación Universitaria Los Libertadores y de la Escuela Superior de Administración Pública -ESAP-. Correo electrónico: pedronelpaez@gmail.com
} 

dinámica comercial y las ventajas comparativas respecto a Venezuela

reveladas (VCR) en términos de contribución al saldo comercial. El uso de esta clase de indicador VCR se justifica por un procedimiento que apunta a revelar mejor las ventajas comparativas ante el sesgo coyuntural significativo que afecta los intercambios con Venezuela en la actualidad.

Palabras clave: dinámica comercial; balance comercial; Colombia; Venezuela; Cuero, Calzado y Marroquinería; ventajas comparativas reveladas; contribución al saldo comercial.

\begin{abstract}
This work studies the dynamics of trade between Colombia and Venezuela in the case of leather, footwear and leather-based industries, in relation to the underlying the comparative-advantage structure. Among the main results, Colombia were able to maintain a trade surplus against Venezuela for the previously mentioned products, despite the economic and political crisis in that country. Leather and footwear are the main products behind this trade surplus, which is confirmed by the calculation of comparative advantages in terms of contribution to the trade balance index. This kind of index is especially suited for revealing comparative advantages when trade short-term fluctuations significantly affect trade, as this is the case for Venezuela nowadays.
\end{abstract}

Keywords: Trade dynamics; Trade balance; Colombia; Venezuela; Leather, footwear and leather-based industries; Revealed comparative advantages; Contribution to the trade balance.

\title{
1. INTRODUCCIÓN
}

Históricamente ha existido una interdependencia comercial entre Colombia y Venezuela.-Por una parte, Venezuela requiere de productos colombianos para abastecer su demanda doméstica. Por otra parte, Colombia depende de Venezuela por ser uno de los principales destinos de sus exportaciones (Gutiérrez y Rosales, 2010). Otro de los factores que explica esta interdependencia es su cercanía geográfica, la cual los ha convertido en socios comerciales históricos, permitiéndoles intercambiar bienes a menores costos de transporte. A esto, se suma la complementariedad productiva, aspectos culturales compartidos y patrones de consumo similares, lo que conforme al modelo gravitacional de comercio dinamiza los intercambios entre los dos socios (Krugman y Obstfeld, 2006; Chaney, 2018).

Las relaciones de los dos países también han estado marcadas por conflictos diplomáticos. Charry (1998), y Beltrán y Afanador (2011) destacan problemas de soberanía en aguas del océano Atlántico, así como el cierre de fronteras por 
la incursión de la guerrilla colombiana en Venezuela (1999), el caso Granda (2005) y el caso Reyes (2008). Asimismo, Romero (2010) señala la relación entre Colombia y Estados Unidos como otra fuente del conflicto diplomático, por las negociaciones del Tratado de Libre Comercio iniciadas en 2004 y el acuerdo militar que permitía establecer bases en Colombia en 2009.

Tales conflictos han ocasionado disminuciones recurrentes en los flujos comerciales bilaterales, haciendo que ambos países trabajen en estrategias de cooperación para enfrentar dicha situación y dinamizar nuevamente el comercio. Así, se evidencian repuntes en los intercambios luego de resolver un conflicto diplomático (Ruiz y Rosales, 2009; Gutiérrez, 2017). Según los términos de Beltrán y Afanador (2011), una de las motivaciones para solucionar los conflictos ha sido recuperar el dinamismo comercial.

Empero y pese a las estrategias de cooperación, en 2006 Venezuela anunció su dimisión de la Comunidad Andina (CAN) y se retiró en 2011, poniendo en riesgo su futuro comercial con Colombia, pues se disolvió el principal escenario de integración regional con ese país. Además de esto, la apertura de Venezuela hacia el Mercado Común del Sur (Mercosur) en 2006 fue concebida como una amenaza para las exportaciones colombianas hacia ese destino (Preusse, 2007). Se hizo entonces necesario establecer un nuevo mecanismo que permitiera conservar las preferencias arancelarias de la CAN y profundizar las relaciones comerciales binacionales (Beltrán y Chávez, 2008). Por esto, en octubre de 2012 se implementó el Acuerdo comercial entre Colombia y Venezuela, denominado «Acuerdo de Alcance Parcial-Comercial N. ${ }^{\circ}$ 28» (AAP.CN'28) en el marco jurídico de la Asociación Latinoamericana de Integración (ALADI) ${ }^{1}$.

Desde la óptica de Colombia, con el AAP.CN²8 se esperaba no solo restablecer las relaciones comerciales con Venezuela sino también incrementar las exportaciones hacia ese destino (MinCIT, 2012). En efecto, el comercio entre ambos países había disminuido un 65\% entre 2009 y 2010, ocasionando la pérdida de participación de Venezuela como segundo destino de las exportaciones colombianas en 2005 al séptimo en $2011^{2}$. Todo esto por los conflictos diplomáticos y el menor ritmo de crecimiento de ambos países en el contexto de la crisis económica mundial (Gutiérrez, 2017).

Pero ¿es posible incrementar las exportaciones hacia un país mediante la firma de un nuevo acuerdo? Al respecto, el aumento de tales flujos depende de la competitividad de los productos ofrecidos (Stellian \& Danna-Buitrago, 2017a).

1 Ver: aladi.org/sitioaladi/acuerdosInfoAcdosAP.html

2 Cálculos de los autores a partir de UNCTADstat (http://unctadstat.unctad.org/EN/) 
Si un producto no dispone de competitividad cuando se implementan espacios comerciales, no podrá responder a la demanda extranjera y sus exportaciones no aumentarán. De hecho, se corre el riesgo de no emprender un Export-Led Growth (Danna-Buitrago, Stellian y Mercado, 2017) y sustituir la producción nacional por las importaciones (Helpman y Krugman, 1985; Sannassee, Seetanah y Jugessur, 2014). La competitividad es entonces una condición sine qua non para aumentar (o al menos mantener) las exportaciones, y la producción nacional.

Precisamente, este artículo estudia la competitividad del sector de Cuero, Calzado y Marroquinería (en adelante sector CCM) entre 1995 y 2017. Primero, porque los productos que lo conforman hacen parte de las corrientes de comercio históricas entre las partes desde 1900 (Urrutia, Posada, Pontón y Martínez, 2000). Segundo, porque están incluidos en el Programa de Transformación Productiva (PTP), una iniciativa del gobierno colombiano implementada en 2009 en pro de la competitividad ${ }^{3}$. Tercero, porque el comercio del sector CCM ha sido completamente liberalizado por el AAP.CNo $28^{4}$.

\section{PROBLEMA DE INVESTIGACIÓN Y REVISIÓN DE LA LITERATURA}

Teniendo en cuenta lo anterior, surgen dos preguntas: ¿Cómo ha sido el posicionamiento del sector CCM en el comercio de Colombia con Venezuela entre 1995 y 2017, y ¿Cómo ha evolucionado la competitividad de tal sector desde el punto de vista de las ventajas comparativas en el mismo lapso? Se señala la pertinencia del primer cuestionamiento, pues permite ver el comportamiento de las exportaciones colombianas del sector CCM en tiempos de crisis venezolana, haciendo posible extraer conclusiones sobre sus fortalezas. De hecho, se trata de uno de los pocos sectores que ha seguido exportando hacia el vecino país después del inicio de la crisis venezolana, la cual empezó a manifestarse en 2009 y terminó por agravarse desde 2013.

3 El PTP señala que los productos CCM: i) disponen de alto potencial exportador por la creatividad, diferenciación y calidad en su diseño; ii) cuentan con vocaciones regionales para su desarrollo que facilitan la generación de procesos de alto valor agregado, innovación y diseño; iii) el sector ha venido creciendo durante la última década y es intensivo en mano de obra por estar conformado por 30.000 empresas formales de insumos, transformación y comercialización. Ver: www.ptp.com.co/ptp-sectores/ historico/cuero-calzado-marroquineria.

4 De las 10008 subpartidas negociadas en el AAP.CN'28, 78 se relacionan a los productos CCM (27 subpartidas para los productos de cuero, 30 para el calzado y 21 para los productos de marroquinería), y todas disponen de una exención del 100\%, o sea, un acceso preferencial de cero aranceles desde 2012. 
Sobre el segundo cuestionamiento, se indica la conveniencia de medir las ventajas comparativas para dar cuenta de la competitividad, pues se entiende por ventaja comparativa la capacidad de fabricar un bien con productividad relativa mayor y diferenciación en sus características. Precisamente, son estos diferenciales de productividad y características los que confieren competitividad. Además, se señala la pertinencia de medirlas mediante un indicador de Ventajas Comparativas Reveladas (VCR) en términos de Contribución al Saldo Comercial (CSC) porque un indicador VCR se calcula mediante datos de comercio internacional (exportaciones e importaciones), y el indicador VCR-CSC permite realizar correcciones del sesgo coyuntural en los intercambios (De SaintVaulry, 2008). Por consiguiente, sus resultados reflejan mejor los cambios de tipo estructural en las (des)ventajas comparativas, y no de tipo coyuntural en razón de la disminución de los flujos comerciales por la crisis venezolana, que coincide con la implementación del acuerdo, lo que conllevaría a errores de interpretación.

En la literatura no existen trabajos que estudien el tema de este artículo. Los trabajos disponibles describen el comercio fronterizo colombo-venezolano (Moncayo, 2003; Mojica y Paredes, 2004) y la importancia de la zona de integración fronteriza (Linares, 2005; Sánchez, 2011). Igualmente, analizan las relaciones comerciales colombo-venezolanas (Gutiérrez y Rosales, 2010; Gutiérrez y Rosales, 2016; Gutiérrez, 2017), la importancia de los acuerdos para solucionar conflictos diplomáticos (Beltrán, 2010; Beltrán y Afanador, 2011), los efectos económico-políticos de la dimisión de Venezuela de la CAN (Malamud, 2006; Casas y Correa, 2007; Preusse, 2007; Beltrán y Chávez, 2008;), y el conflicto diplomático entre los dos países (Palotas, 2008).

Existen algunos trabajos que se interesan en la competitividad del calzado, del cuero o de la marroquinería, mediante un enfoque diferente al de las ventajas comparativas. Villegas y Zapata (2007) estudian la competitividad del cuero y calzado colombiano respecto a la CAN mediante indicadores de comercio exterior. Romero, Monroy y Ramírez (2017) analizan la competitividad de 32 empresas de calzado de Cúcuta mediante el Diamante de Porter (1990). Perdomo y Malaver (2002) estudian los costos de producción y los niveles de diferenciación del calzado para dama en Norte de Santander, pero no lo hacen desde la perspectiva de las ventajas comparativas; es decir, no calculan un indicador VCR que halle diferenciales de productividad y características entre un país y otro(s).

Tampoco existen investigaciones que revelen las ventajas comparativas centrándose simultáneamente en i) una nomenclatura exhaustiva de productos CCM; ii) el AAP.CN²8; iii) el indicador VCR-CSC aquí propuesto. Echavarría (1995) revela ventajas comparativas de productos colombianos en Venezuela, Chile y 
México, pero no especifica los periodos ni tampoco se focaliza en los productos CCM. García y Maldonado (2013) calculan ventajas comparativas del calzado de cuero colombiano, e indicadores básicos de comercio internacional, respecto a Estados Unidos entre 1980 y 2008, sin interesarse en el mercado venezolano. Además, los dos trabajos señalados utilizan los indicadores de ventajas comparativas propuestos por Balassa $(1965,1986)$, pese a sus limitaciones ${ }^{5}$.

Así las cosas, este artículo se divide en cinco secciones; la primera es la introducción y la segunda, la presentación del problema de investigación. La sección 3 presenta la estrategia metodológica para analizar la dinámica comercial del sector CCM respecto a Venezuela y calcular sus VCR-CSC, al igual que el marco teórico. Asimismo, presenta las razones de la elección de tal indicador. La sección 4 da cuenta de los resultados en términos de dinámica comercial y VCR-CSC para 1995-2017. Finalmente, la sección 5 enuncia conclusiones.

\section{ESTRATEGIA METODOLÓGICA Y MARCO TEÓRICO}

\subsection{Dinámica comercial del sector CCM entre Colombia y Venezuela}

Se analiza la dinámica comercial del sector CCM entre Colombia y Venezuela, para determinar si las exportaciones colombianas aumentaron más que proporcionalmente respecto al incremento de las importaciones provenientes de Venezuela entre 1995 y 2017, y así responder el primer cuestionamiento que motiva esta investigación. Esto permite abordar otro interrogante: ¿El superávit comercial, en caso de existir, se ha mantenido después del inicio de la crisis económica venezolana? Así, se introducen pistas sobre las razones que originan la capacidad del sector de seguir exportando hacia Venezuela en tiempos de crisis, lo que representaría la excepción y no la regla, pues las exportaciones colombianas de otros sectores son actualmente inexistentes.

Para ello se utilizan estadísticas de comercio internacional de la UNCTADstat para grupos de productos CCM según la Clasificación Uniforme para el Comercio Internacional (CUCI), Revisión 3, a tres dígitos, pues esta nomenclatura: i) se emplea desde 1950 por la ONU para compilar estadísticas sobre comercio internacional de productos; ii) es conocida como instrumento de estudio de las tendencias a largo plazo del comercio internacional de productos; iii) reúne los productos en grupos de manera uniforme a nivel mundial, facilitando los análisis económicos y las comparaciones.

5 Ver: Stellian y Danna-Buitrago (2017a; 2017b) para una explicación sobre las limitaciones mencionadas. 
Con base en lo anterior, el análisis se focaliza en ocho categorías CUCI, las cuales se relacionan a los productos CCM que conforman precisamente el sector:

[211] Cueros y pieles (Excepto pieles finas) sin curtir.

[212] Pieles finas sin curtir (Incluso cabezas, colas, patas y otras piezas o cortes adecuados para peletería). Excepto las pieles y los cueros del grupo 211.

[611] Cuero.

[612] Manufacturas de cuero natural o sintético, n. e. p.; artículos de talabartería y guarnicionería.

[613] Pieles finas curtidas o adobadas (Incluso cabezas, colas, patas y otras piezas o recortes), ensamblados o sin ensamblar (sin agregados de otros materiales).

[831] Baúles, neceseres, maletas, maletines para documentos, carteras o portafolios, maletas escolares, estuches para aparatos fotográficos, entre otros.

[848] Prendas y accesorios de vestir que no sean de materias textiles, sombreros y otros artículos de tocado de todo tipo de materiales ${ }^{6}$.

[851] Calzado.

En tal sentido, se identifica que el Cuero está conformado por las categorías [211], [212], [611], [612] y [613]; el calzado por la categoría [851]; y la marroquinería por las categorías [831] y [848].

\subsection{Ventajas comparativas reveladas del sector colombiano CCM frente a Venezuela en términos de contribución al saldo comercial}

Como se mencionó antes, el concepto de ventaja comparativa hace referencia a la capacidad de fabricar un bien con productividad relativa mayor y diferenciación en sus características, lo que le confiere competitividad en una zona de

6 Entre las ocho categorías, la 848 es la única que no contiene exclusivamente productos CCM. Esta incluye las prendas y accesorios de vestir de cuero, los artículos de peletería, pero también las prendas y accesorios de vestir de materiales plásticos y caucho vulcanizado. Así, el análisis de esta categoría solo representa una aproximación para los productos CCM correspondientes. 
intercambios. En este orden de ideas, este trabajo no concibe el concepto de la ventaja comparativa según la idea de Ricardo (1817), quien se focalizó en los costos relativos de fabricar productos homogéneos que determinan los precios relativos de venta (Cho y Moon, 2002; Anca, 2012). En contraste, la versión moderna del concepto incluye aspectos más allá de dichos costos, tomando en cuenta los trabajos de Lafay (1987), Porter (1990), Chevassus-Lozza y Gallezot (1995), Eaton y Kortum (2002), Costinot (2009), Chor (2010) y Cuñat y Melitz (2012). Bajo esta perspectiva, se admite que los determinantes de las ventajas comparativas conciernen a varios aspectos y no únicamente a la productividad relativa mayor, dando origen a un concepto más flexible de ventajas comparativas (ver Stellian y Danna-Buitrago, 2017a, Páez, Jímenez y Danna-Buitrago, 2018).

Las Ventas Comparativas se calculan a partir del método estándar, tomando en cuenta los intercambios de un país $i$, respecto a un producto $k$, en un periodo $t$, y en una zona de intercambios. Se calcula un indicador que da cuenta de manera sintética del nivel de ventajas comparativas para cualquier $(i ; k ; t)$ en la zona considerada. Las Ventajas Comparativas se revelan entonces por los flujos comerciales como lo propuso Balassa (1965), por lo que se acuña el término de Ventajas Comparativas Reveladas (VCR). Así, se admite que los flujos comerciales reflejan indirectamente las ventajas comparativas y que estas reflejan los factores que las determinan (Balassa, 1977; Lafay, 1987; Stellian y DannaBuitrago, 2017a).

Se elige el indicador VCR en términos de Contribución al Saldo Comercial (CSC), un indicador poco utilizado pese a su superioridad empírica (a) y teóri$\mathrm{ca}(\mathrm{b})$ respecto a otros indicadores VCR representativos en la literatura como Balassa (1965), Balassa (1986), Hoen y Oosterhaven (2006), Yu, Cai y Leung (2009) y Laursen (2015).

a. En términos empíricos. Danna-Buitrago (2017) efectuó pruebas de minimización de la desviación típica en el tiempo de cada uno de los indicadores arriba enunciados, lo cual le permitió concluir que el indicador VCR-CSC muestra las mejores propiedades en términos de estabilidad en el tiempo. Tal estabilidad es necesaria para que un indicador VCR sea consistente con la teoría de las ventajas comparativas, pues estas tienden a cambiar solo en horizontes de largo plazo, de modo que un indicador no debe tender a cambiar mucho de un período al siguiente.

b. En términos teóricos. Ninguno de los indicadores VCR arriba señalados combina estas propiedades: i) incluir en su concepción las exportaciones e importaciones, y por ende considerar el comercio inter-rama e intra-rama; ii) disponer de un protocolo de ajuste de los flujos comerciales para limitar 
el sesgo generado por las fluctuaciones coyunturales al revelar las ventajas comparativas; iii) vincular las (des)ventajas comparativas al tamaño de la economía nacional mediante una normalización por el producto interno bruto (PIB); iv) incluir el comercio de $k$ en toda la zona considerada. Precisamente, el indicador VCR-CSC combina estas propiedades, permitiendo calcular las ventajas comparativas de forma más consistente (Danna-Buitrago, 2017; Stellian y Danna-Buitrago, 2017a).

A continuación, se presenta el indicador VCR-CSC y las variables que lo componen:

$$
\operatorname{CSC}_{i k t}:=\frac{1000}{Y_{i t}}\left[X_{i k t}^{\prime}-M_{i k t}^{\prime}-w_{k t}\left(X_{i t}^{\prime}-M_{i t}^{\prime}\right)\right]
$$

donde $C S C_{i k t}$ es el indicador VCR-CSC para el país $i$ en $t$ respecto al producto $k$. El cálculo del indicador se hace en 5 etapas:

1. Calcular $W_{k t}:=\sum_{i=1}^{n}\left(X_{i k t}+M_{i k t}\right)$ para cada $\langle k, t\rangle$, es decir, el comercio de $k$ en $t$ efectuado por todos los países de la zona, y $W_{t}:=W_{1 t}+W_{2 t}+\cdots W_{K t}$ para cada $t$, que representa el comercio de todos los productos en $t$, realizado por los países de la zona.

2. Calcular $w_{k t}:=\frac{w_{k t}}{w_{t}}$ para cada $\langle k, t\rangle$. Esta variable es el peso en $t$ de $k$ en el comercio total de $K$ productos dentro de la zona de intercambios considerada.

3. Calcular $X_{i k t}^{\prime}-M_{i k t}^{\prime}:=\frac{w_{k r}}{w_{k t}}\left(X_{i k t}-M_{i k t}\right)$ que consiste en ajustar el saldo comercial $X_{i k t}-M_{i k t}$ asociado a $\langle i, k, t\rangle$ por $\frac{w_{k r}}{w_{k t}}$. Esta operación hace que en cada período los flujos comerciales de $k$ resulten en el mismo peso de $k$ para cualquier $t$, mientras que dicho peso corresponde al peso de un período de referencia denotado $r$.

4. Calcular la diferencia entre $X_{i t}^{\prime}-M_{i t}^{\prime}:=\sum_{k=1}^{K}\left(X_{i k t}^{\prime}-M_{i k t}^{\prime}\right)$ y $w_{k t}\left(X_{i t}^{\prime}-\right.$ $M_{i t}^{\prime}$ ). La primera variable es el saldo comercial efectivo ajustado de $i$ en $k$ en $t$ y la segunda variable es el saldo comercial total ajustado de $i$ en $t$ ponderado por el peso de $k$ en el comercio total de de la zona en $t$. Esta segunda variable es el saldo comercial teórico ajustado de $i$ en $k$ en $t$ que revela la ausencia de ventajas y desventajas.

5. Normalizar dicha diferencia en milésimas del PIB $\left(\frac{1000}{Y_{i t}}\right)$. 
En este contexto y para el caso del presente artículo, ajustar los flujos comerciales (etapa 3) permite que los valores de las exportaciones e importaciones no reflejen tanto las disminuciones puntuales ocasionadas por la crisis económica venezolana, que comenzó a manifestarse en 2009 y se agravó en 2013, la cual responde en gran medida al enfoque con el que el gobierno de Nicolás Maduro ha enfrentado los desafíos económicos (Vera, 2018). De esta manera, no se desvía el análisis de la competitividad, ya que las variaciones en el indicador VCR-CSC dependerán más de los cambios estructurales y menos de los cambios coyunturales.

El indicador se calculó tomando como periodo de referencia el año 2008 para ajustar los flujos comerciales porque:

1. Como se muestra en la gráfica 1 , los intercambios de mercancías entre Colombia y Venezuela se redujeron drásticamente luego del 2008. Precisamente, se registra un aumento continuo en las exportaciones de Colombia hacia Venezuela entre 2003 y 2008, antes de disminuir de manera casi continua en los siguientes años. Las importaciones también disminuyen a partir de 2009, después de una relativa estabilidad desde 1995.

2. La evolución en los intercambios antes mencionada se atribuye a la inestabilidad que empezó a afectar la economía venezolana en $2008^{7}$. Dicha inestabilidad se observa mediante la tasa de variación del PIB real (ver gráfica 1), la cual en 2009 vuele a ser negativa, siendo 2003 el último año en el que se presentó una tendencia similar. Luego de una breve recuperación entre 2010 y 2013, la tasa de variación del PIB volvió a caer y sigue siendo negativa hasta el día de hoy.

3. En consecuencia, a partir de 2009 los intercambios se ven afectados por fluctuaciones coyunturales significativas que limitan su capacidad para revelar las ventajas comparativas. Por esto, no se debería utilizar un año entre 2009 y 2017 para ajustar los flujos comerciales, con el ánimo de limitar el sesgo en la medición indirecta de las ventajas comparativas mediante los intercambios. Se selecciona entonces el 2018 por ser el último año antes de la crisis económica que afecta Venezuela.

Al final, si $\left(X_{i k t}^{\prime}-M_{i k t}^{\prime}\right)-\frac{W_{k t}}{W_{t}}\left(X_{i t}^{\prime}-M_{i t}^{\prime}\right)>0$ se constata la existencia de ventajas comparativas de $i$ para $k$ en $t$ (saldo efectivo mayor al saldo teórico); y viceversa.

7 Sobre los factores económico-políticos externos e internos de la crisis en Venezuela, ver Lampa (2016). 


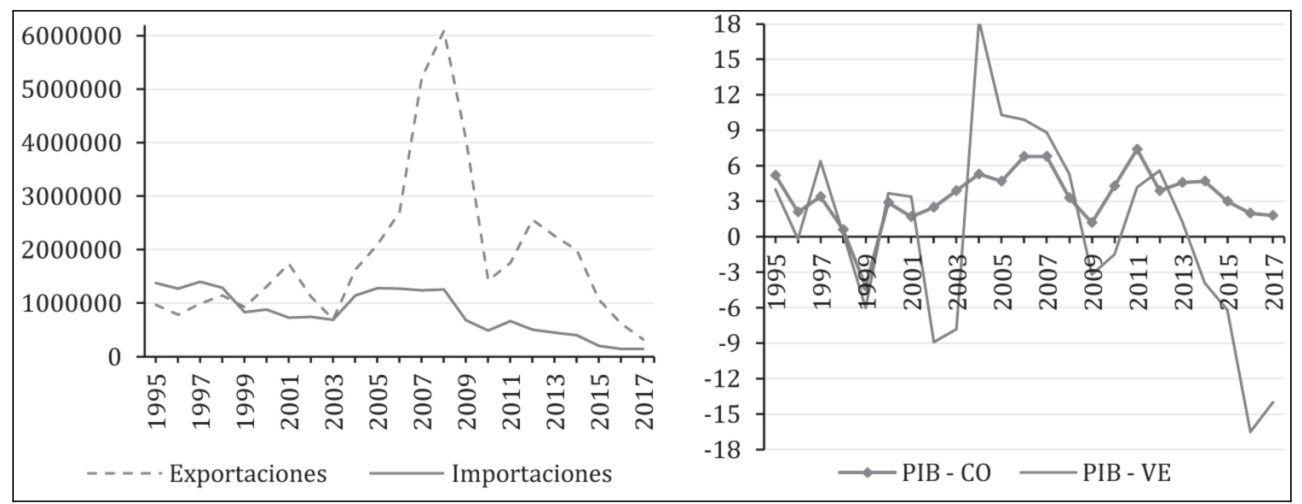

Fuente: UnctadSTAT, World Economic Outlook, cálculos propios.

Gráfica 1. Exportaciones e importaciones de mercancías de Colombia con Venezuela (miles de dólares), y tasa de variación del PIB real (\%), 1995-2017.

\section{RESULTADOS: DINÁMICA COMERCIAL Y VCR-CSC DEL SECTOR CCM}

Las exportaciones del sector CCM hacia Venezuela han sido fluctuantes entre 1995 y 2017. Como se observa en la gráfica 2, durante el período previo al AAP.CN 28 , la tasa de crecimiento anual fue positiva en la mayoría de los años, 10 sobre los 17 . Por el contrario, durante los años posteriores al AAP.CN 28 la tasa de crecimiento fue negativa para todos los períodos. Así, la tasa promedio de crecimiento pre-AAP.CNo28 (1995-2012) fue de 38\%, mientras que el promedio post-AAP.CN 28 (2013-2017) fue de $-28 \%$, lo que significa

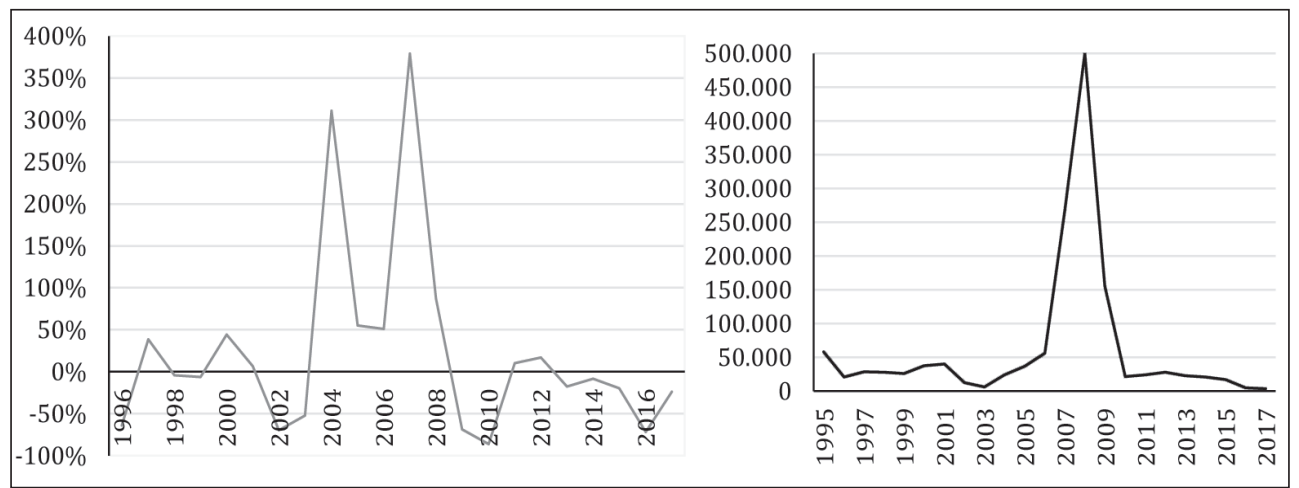

Fuente: UnctadSTAT, cálculos propios.

Gráfica 2. Tasa de crecimiento, exportaciones de productos CCM hacia Venezuela, y exportaciones del sector CCM hacia Venezuela (miles de dólares), 1996-2017. 
para Colombia el incumplimiento de uno de los propósitos del acuerdo: incrementar sus exportaciones hacia ese destino.

Asimismo, las exportaciones promedio de CCM hacia Venezuela pre-AAP.CNo28 fueron de 76103 miles de dólares, mientras que el promedio post-AAP.CN 28 fue de solo 13847 miles de dólares; es decir, una disminución aproximada del $82 \%$. De hecho, ni siquiera el anuncio de dimisión de la CAN de Venezuela en 2006 ni su salida en 2011 impactaron así dichas exportaciones, pues en 2006 se exportaron 55834 miles de dólares y en 2011 esta cifra se ubicó en 23950 miles de dólares.

La desaceleración del crecimiento de las exportaciones colombianas de CCM hacia Venezuela después del AAP.CN'28 responde a otros elementos y no a la implementación del acuerdo, pues este instauró medidas para facilitar los intercambios (y no lo contrario). Entre dichos elementos se destacan las decisiones de política económica de Venezuela, las cuales introdujeron vulnerabilidades que afectan el volumen de los intercambios de todos los productos (y no únicamente de CCM) y en relación con todos los países (y no solamente respecto a Colombia).

La dependencia venezolana de las exportaciones de petróleo constituye un claro ejemplo de vulnerabilidad, pues el ingreso petrolero se convirtió desde 1930 en el principal eje dinamizador del país (Melcher, 1995; García y Reyes, 2008). De hecho, Venezuela se transformó en una economía altamente especializada en extracción/exportación de petróleo en detrimento de la agricultura y la industria, de modo que comenzó a importar, sirviéndose de las divisas del petróleo, bienes de consumo de primera necesidad y de lujo, al igual que materias primas intermedias y procesadas, maquinaria y vehículos (Hernández, 2009).

Al desplomarse los precios del petróleo en 2015, y por ende los ingresos de origen petrolero, se agrava la crisis y la imposibilidad de importar ${ }^{8}$. En efecto, en 1997 las divisas del petróleo representaron 87\% de los ingresos totales de divisas del Banco Central de Venezuela, mientras que en 2015 solo el 44\%, pasando de 18323 millones de dólares en 1997 a 9585 en 2015, lo que coincide con la baja del precio internacional del petróleo de 96,29 dólares por barril en 2014 a 49,51 en 2015.

En consecuencia, «en los últimos 4 años (...) el gobierno de Maduro ha recortado las importaciones (en todo su espectro de bienes y servicios) en casi $76 \%$ »

8 Esto constituye uno de los factores que originan la crisis venezolana. Sobre los factores estructurales que condicionaron la economía del petróleo y contribuyeron al advenimiento de la crisis, ver Vera (2018). 
(Vera, 2018, p.84). En tal sentido, las importaciones venezolanas desde Colombia pasaron de 2169515 miles de dólares en 2013 a 448221 en 2017, disminuyendo el $79 \%$. Y sus importaciones desde el mundo pasaron de 44951787 miles de dólares en 2013 a 9762288 en 2017, presentando una baja del 78\%. Asimismo, las importaciones venezolanas de CCM desde Colombia pasaron de $25037 \mathrm{mi}-$ les de dólares en 2013 a 6234 en 2017, reduciéndose en un 75\%. Y sus importaciones de CCM desde el mundo disminuyeron $71 \%$, pasando de 497299 miles de dólares en 2013 a 143436 en 2017. En síntesis, la reducción en las importaciones no solo ha perjudicado el sector CCM, sino también todas las importaciones realizadas por Venezuela desde Colombia y el mundo.

No obstante y pese a que el crecimiento de las exportaciones CCM es negativo para todos los periodos post-AAP.CN ${ }^{\circ} 28$, cabe resaltar que se han seguido exportando ese tipo de productos hacia Venezuela aún durante las crisis ${ }^{9}$. Además, los valores en millones de dólares de las exportaciones colombianas CCM han sido similares a los registrados durante los años pre-AAP.CN ${ }^{\circ} 28^{10}$ (ver gráfica 2). Ciertamente, en algunos periodos no se alcanzan los niveles pre-crisis, pero al menos se han mantenido las exportaciones; contrario, por ejemplo, a lo registrado por el «Queso y cuajada» y los «Jugos de frutas y vegetales, sin fermentar, sin alcohol», cuyas exportaciones hacia Venezuela cesaron desde 2013.

En cuanto a las importaciones colombianas del sector CCM procedentes de Venezuela, la gráfica 3 muestra que decrecieron de manera casi continua desde 1995. En efecto, hubo una reducción significativa entre 1995 y 2003, y luego una disminución continua, hasta llegar a representar apenas 146 mil dólares en 2017. Esta tendencia se constata durante más de 20 años, extendiéndose más allá del período de crisis económica venezolana. El bajo volumen de las importaciones hace entonces que la balanza comercial del sector CCM registrada por

9 Esto pese a i) la disminución del 31,9\% de su PIB en 2017 respecto a 2013; ii) la inflación originada en 2012 que se ha vuelto hiperinflación desde finales de 2017; iii) la falta de ingresos, insumos, materias primas y bienes de capital importados en las empresas; iv) el colapso del mecanismo de control de cambios desde septiembre 2017, dejándolos a expensas de un mercado paralelo ilegal, con el cual la tasa de cambio se disparó; v) la depreciación significativa del bolívar entre enero y noviembre 2017; vi) la escasez de divisas por el manejo deficiente de ingresos petroleros, la pérdida de acceso a los mercados financieros externos y la política macroeconómica (CEPAL, 2018; Vera, 2018).

10 Los valores exportados en 2007, 2008 y 2009 constituyen una excepción, pues son el resultado de la recuperación de Venezuela debido al alza de los precios internacionales del petróleo que le permitió tener un excedente de divisas y así aumentar sus importaciones. Ver: https://es.statista.com/estadisticas/635114/precio-medio-delcrudo-fijado-por-la-opep/ 
Colombia frente a Venezuela sea casi igual a las exportaciones, tal como lo muestra la gráfica 3 .

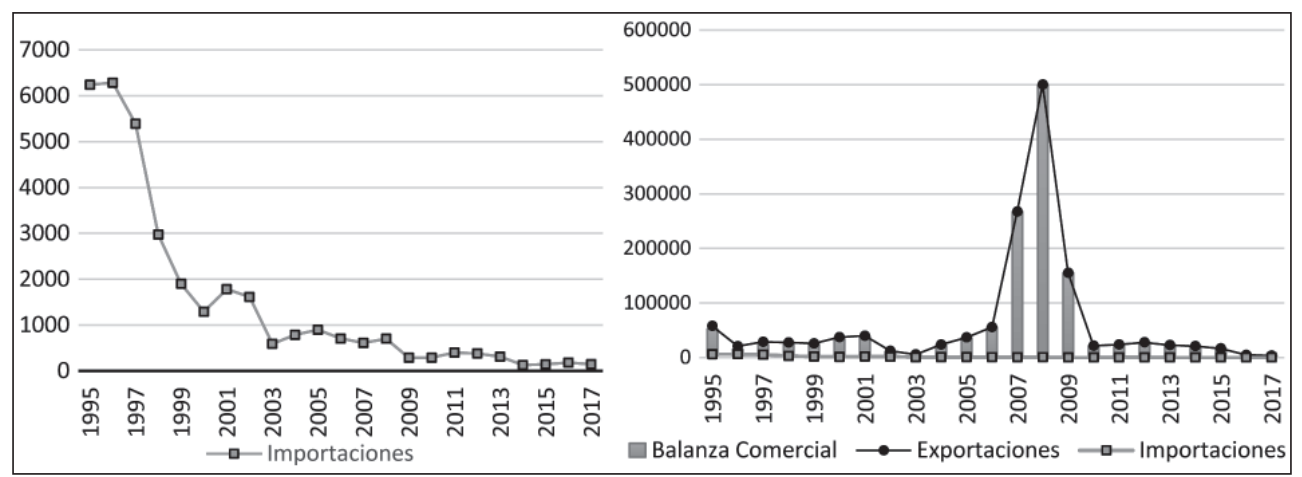

Fuente: UnctadSTAT, cálculos propios.

Gráfica 3. Importaciones del sector CCM desde Venezuela, y balanza comercial del sector CCM respecto a Venezuela (miles de dólares), 1995-2017.

Al combinar la evolución de las exportaciones y las importaciones, se constata que:

1. Las exportaciones tendieron a aumentar y tal incremento habría podido continuar si la economía venezolana no hubiera enfrentado una de las peores crisis económicas de la historia latinoamericana. Sin embargo, aún en situación de crisis, Colombia sigue exportando los productos CCM hacia Venezuela.

2. El nivel de las importaciones ha sido bajo aun antes de la crisis, y continúa siéndolo hoy.

3. En consecuencia, la balanza comercial es excedentaria y su nivel habría podido ser mayor sin la crisis económica en Venezuela.

Por esta razón, se puede conjeturar que los productos que conforman el sector CCM constituyen un punto fuerte de Colombia en el comercio con su socio venezolano. En otras palabras, Colombia tiene la capacidad de producir a menores costos relativos (mayor productividad) y mayor diferenciación en comparación con Venezuela. Bajo esta óptica, se debe validar o invalidar tal conjetura mediante la presentación de los resultados de los cálculos de ventajas comparativas.

Previamente, la gráfica 4 permite estudiar en detalle las ocho categorías de productos CCM según la CUCI, que conforman el sector CCM. Esto, con el fin 


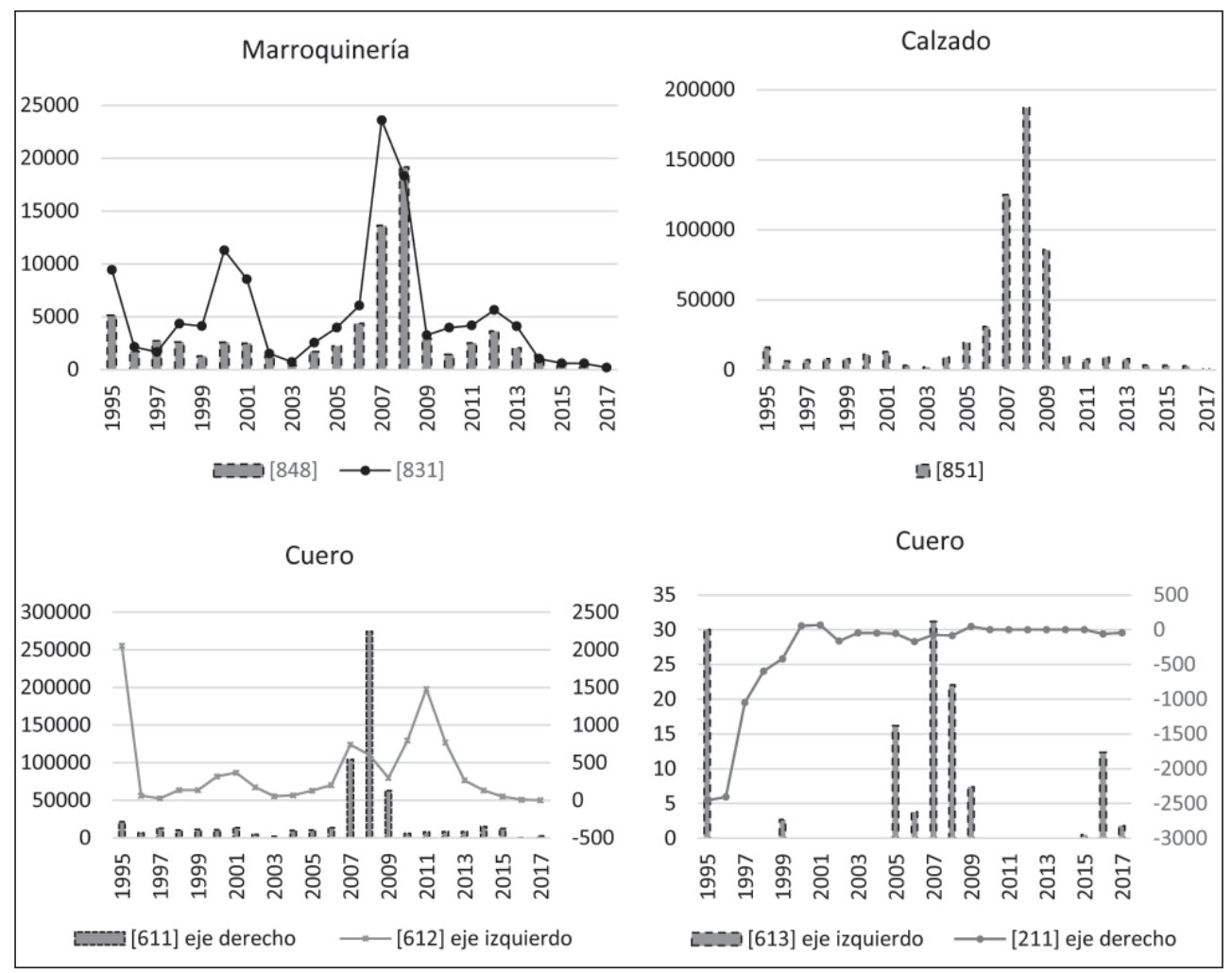

Fuente: UnctadSTAT, cálculos propios.

Gráfica 4. Importaciones de productos CCM desde Venezuela, y balanza comercial de productos CCM respecto a Venezuela (miles de dólares), 1996-2017.

de ver cuáles categorías tuvieron el mayor impacto sobre la balanza comercial de dicho sector.

Nota: La categoría 212 "Pieles finas sin curtir" no se ha incluido en las gráficas del grupo "cuero", pues a excepción del año 1996 en donde la balanza comercial se situó en -9 , esta se ha mantenido en 0 .

En este orden de ideas, la gráfica 4 muestra lo siguiente:

a. Solo en el caso de la categoría 211 «Cueros y pieles (Excepto pieles finas) sin curtir» se observa una balanza comercial negativa en los primeros años, antes de una reducción de este déficit, así que dicha balanza se mantiene alrededor de cero a partir de 2000 . 
b. Para las siete otras categorías, la balanza comercial siempre ha sido positiva (a excepción de la categoría 212 en 1996), sobre todo en el caso de las categorías 851 «Calzado» y 611 «Cuero».

c. En este sentido, todas las categorías, salvo una, contribuyen a la conformación de un excedente de la balanza comercial del sector CCM; y dos contribuyen de manera decisiva (calzado «851» y cuero «611»).

Lo anterior es confirmado por el análisis del indicador VCR-CSC, el cual permite constatar que el cuero y el calzado registran las ventajas comparativas más altas, tendiendo a mantenerse alrededor de 0,28 y 0,15 respectivamente entre 1995 y 2008, antes de presentar una tendencia a disminuir a partir de 2009 (ver gráfica 5). Sin embargo, esta tendencia a la baja aparece al mismo tiempo que la inestabilidad económica que afecta Venezuela, por lo que se debería tomar con precaución. En efecto, a pesar del ajuste realizado en los flujos comerciales tomando como base el 2008, las exportaciones y las importaciones entre ambos países pueden seguir conservando un sesgo coyuntural, sobre todo en un periodo de crisis económica grave. En este sentido, el ajuste permite limitar, pero no eliminar, dicho sesgo, por lo que los análisis deberían centrase en los datos del indicador VCR-CSC entre 1995 y 2008. Bajo esta perspectiva, es posible afirmar que Colombia dispone de las ventajas comparativas más fuertes frente a Venezuela en el cuero y el calzado, lo que permite hallar un primer resultado: estos dos productos tienen el mayor potencial, en comparacióncon los demás productos CCM, para contribuir a la conformación de excedentes comerciales de Colombia frente a Venezuela mediante tales productos.

Los análisis permiten ver que hay otras dos categorías que presentan un indicador VCR-CSC de nivel intermedio: 831 «Baúles, neceseres, maletas, maletines para documentos, carteras o portafolios, maletas escolares, estuches para

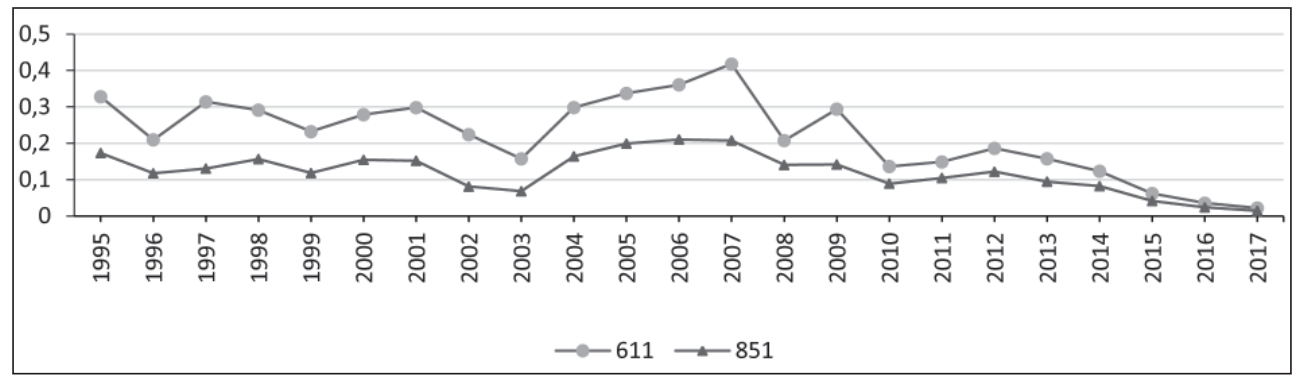

Fuente: cálculos propios.

Gráfica 5. Indicador de ventajas comparativas reveladas, Colombia frente a Venezuela, Cuero (611) y Calzado (851), 1995-2017 (\%o PIB colombiano). 
aparatos fotográficos, entre otros» y 848 «Prendas y accesorios de vestir que no sean de materias textiles, sombreros y otros artículos de tocado de todo tipo de materiales» (ver gráfica 6).

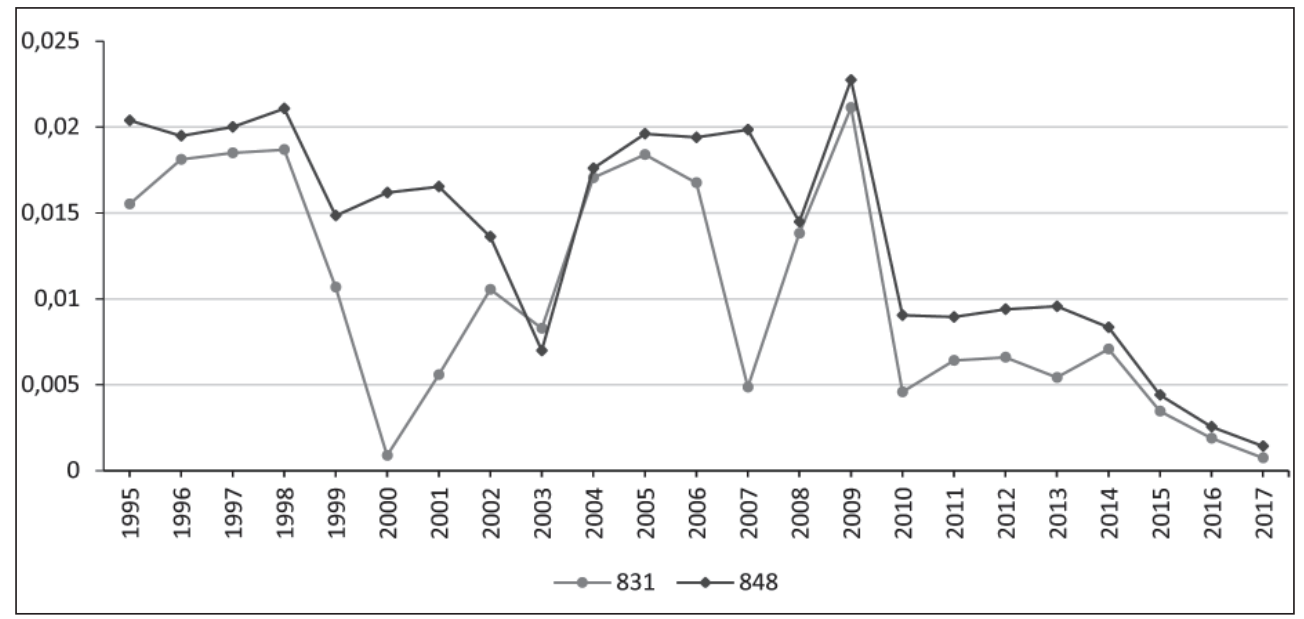

Fuente: cálculos propios.

Gráfica 6. Indicador de ventajas comparativas reveladas, Colombia frente a Venezuela, categorías 831 y 848, 1995-2017 (\%o PIB colombiano).

Nuevamente, la tendencia del indicador VCR-CSC a disminuir a partir de 2009 debería tomarse con precaución, y focalizarnos en los resultados del período 1995-2008. Precisamente, es durante este período que el indicador VCR-CSC revela ventajas comparativas para las dos categorías de productos antes señaladas, aunque son ventajas menores si se comparan con las ventajas registradas por el cuero (611) y el calzado (851). Por consiguiente, se halla un segundo resultado: los artículos incluidos en las categorías 831 y 848, es decir los artículos de viaje y las prendas, accesorios de vestir hechos en cuero, no tienen tanto potencial como el cuero y el calzado para contribuir a la conformación de excedentes comerciales de Colombia frente a Venezuela.

Igualmente, en la gráfica 7 se puede observar que una categoría de productos presenta un indicador VCR-CSC muy cerca de cero, positivo en algunos años y negativo en otros. Se trata de la categoría 612 «Manufacturas de cuero natural o sintético, n. e. p.; artículos de talabartería y guarnicionería». Por su parte, la categoría 211 «Cueros y pieles (Excepto pieles finas) sin curtir» presenta un indicador VCR-CSC igual a cero o negativo en algunos años, pero muy cercano de cero. Y para terminar, las categorías 212 «Pieles finas sin curtir» y 613 «Pieles finas curtidas o adobadas» presentan un indicador VCR-CSC igual o casi igual a cero. 

dinámica comercial y las ventajas comparativas respecto a Venezuela

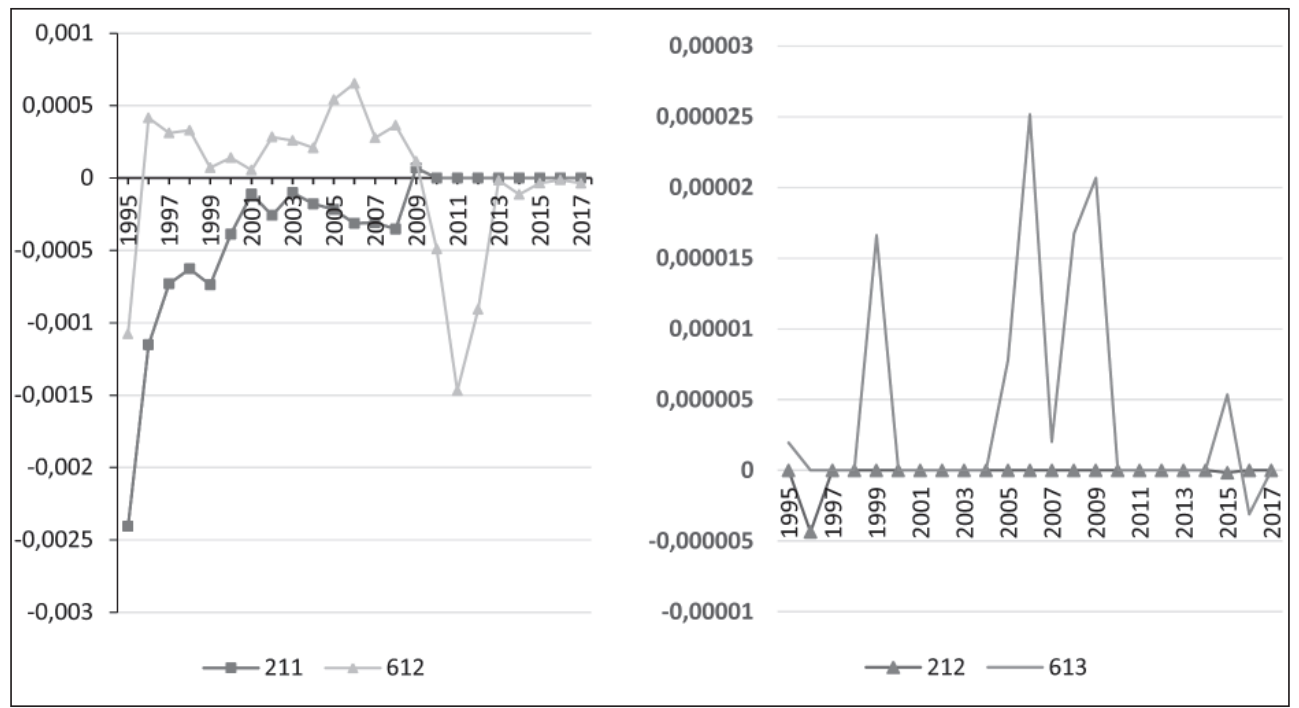

Fuente: cálculos propios.

Gráfica 7. Indicador de ventajas comparativas reveladas, Colombia frente a Venezuela, categorías 211, 612, 212 y 613, 1995-2017 (\% PIB colombiano).

En consecuencia, las cuatro categorías antes mencionadas no disponen de ventajas comparativas significativas, y por esta razón, no tienen el potencial para contribuir a la conformación de excedentes comerciales de Colombia frente a Venezuela mediante los productos CCM. Empero, no se trata tampoco de desventajas comparativas significativas, lo que lleva a un tercer resultado: ninguna categoría de productos CCM presenta desventajas comparativas significativas, lo que impediría que Colombia aproveche el comercio con Venezuela para registrar excedentes comerciales a partir de estos productos. En el peor de los casos, como ya se ha señalado, las desventajas comparativas son débiles.

\section{CONCLUSIÓN}

Las relaciones comerciales entre Colombia y Venezuela han estado marcadas por la grave crisis económica que afecta al segundo país. Sin embargo, Colombia pudo mantener un cierto nivel de exportaciones hacia Venezuela para los productos de Cuero, Calzado y Marroquinería (CCM). Esto sugiere que tales productos constituyen un punto fuerte de Colombia en sus intercambios con el vecino país, lo que se confirma por el bajo nivel de importaciones que existía aún antes de la crisis y una balanza comercial de productos CCM que siempre 
ha sido excedentaria entre 1995 y 2017. El cálculo y análisis de las ventajas comparativas, reveladas a partir de un indicador en términos de contribución al saldo comercial (CSC), también sugiere que los productos CCM disponen de oportunidades sostenibles de ventas en el mercado venezolano, sobre todo el cuero y el calzado. En este sentido, en caso de que Venezuela pueda encontrar una salida a la crisis económica, sería posible reconstruir sus relaciones comerciales con Colombia a partir de los intercambios de productos CCM. Esto requiere que Venezuela deba orientarse hacia una nueva regulación de sus dinámicas macroeconómicas.

La presente investigación podría replicarse en el marco de otros productos, y así ampliar los conocimientos respecto a los esquemas de especialización internacional entre Colombia y Venezuela. Nuevamente, esto apunta a identificar las tendencias de largo plazo -más allá del contexto actual de crisis económica en Venezuela- en el comercio entre ambos países, al igual que la estructura subyacente de ventajas comparativas. Posiblemente, existan otros productos con características similares a los productos CCM, es decir, productos que mostraron una cierta resistencia frente a la crisis económica que afecta al socio histórico de Colombia. Esto podría entonces dar lugar a una reflexión según la cual las entidades públicas de ambos países deberían trabajar en pro de la recuperación de los niveles anteriores de comercio, con base en aquellos productos que representan los puntos fuertes de Colombia, incluyendo los productos CCM.

\section{REFERENCIAS}

Anca, H. (2012). Literature Review of the Evolution of Competitiveness Concept. Annals of Faculty of Economics, University of Oradea, 1(1), 41-46.

Balassa, B. (1965). Trade liberalization and 'revealed' comparative advantage. Manchester School, 33(2), 99-123.

Balassa, B. (1977). 'Revealed' comparative advantage revisited: An analysis of relative export shares of the industrial countries 1953-1971. The Manchester School, 45(4), 327-344.

Balassa, B. (1986). Comparative advantage in manufactured goods: a reappraisal. The Review of Economics and Statistics, 315-319.

Beltrán, N. \& Chávez, M. (2008). Determinantes de la integración colombo-venezolana y perspectivas ante la dimisión de Venezuela en la CAN. Equidad y desarrollo (9), 47-59. 
Beltrán, N. (2010). Integración económica Colombo Venezolana: Dinámica en medio de la incertidumbre política y el regionalismo abierto en el hemisferio. Global journal of human social science, 10(6), 18-31.

Beltrán, N. \& Afanador, B. (2011). Colombia y Venezuela: a repensar los acuerdos económicos para relanzar la integración bilateral. Administración y Desarrollo, 53(39), 123-136.

Casas, A. \& Correa, M. E. (2007). ¿Qué pasa con la Comunidad Andina de NacionesCAN? Papel político, 22(2), 591-632.

Chaney, T. (2018). The Gravity Equation in International Trade: An Explanation. Journal of Political Economy, 126(1), 150-177.

Charry, H. (1998). Propuestas diplomáticas y políticas, en Venezuela y Colombia en el nuevo milenio. Caracas: CAF-Banco de Desarrollo de América Latina.

Chevassus-Lozza, E. \& Gallezot, J. (1995). La compétitivité hors-prix dans les échanges de produits agricoles et agro-alimentaires français sur le marché communautaire, Économie et prévision, 117(1), 143-154.

Chor, D. (2010). Unpacking sources of comparative advantage: A quantitative approach. Journal of International Economics, 82(2), 152-167.

Cho, D. \& Moon, H. (2002). From Adam Smith to Michael Porter: Evolution of Competitiveness Theory Singapore: World Scientific.

Costinot, A. (2009). On the origins of comparative advantage. Journal of International Economics, 77(2), 255-264.

Cuñat, A. \& M. Melitz (2012). Volatility, labor market flexibility, and comparative advantage, Journal of the European Economic Association, 10(2).

Danna-Buitrago, J. P. (2017). La Alianza del Pacífico+ 4 y la especialización regional de Colombia: una aproximación desde las ventajas comparativas. Cuadernos de Administración, 30(55), 163-192.

Danna-Buitrago, J. P. Stellian, R., Mercado, A. (2017). Términos y expectativas de la liberalización comercial entre Colombia y Estados Unidos. En J. P. Danna-Buitrago. (Ed.), Análisis retrospectivo de la integración internacional del sector manufacturero colombiano en el marco del Tratado de Libre Comercio con Estados Unidos (pp. 35-42). Bogotá: Los Libertadores.

De Saint-Vaulry, A. (2008). Base de données CHELEM-commerce international du CEPII, Document de travail CEPII, 9, Centro de información y estudios prospectivos internacionales. 
Eaton, J. \& Kortum, S. (2002). Technology, geography, and trade, Econometrica, 70(5), 1741-1779.

Echavarría, J. (1995). El G3 en el camino de la integración continental. Coyuntura Económica, 25(3), 111-137.

García, M., \& Reyes, R. (2008). Análisis de la Política Económica en Venezuela, 1998-2006. Oikos: Revista de la Escuela de Administración y Economía, (26), 25-47.

García, R., \& Maldonado, A. (2013). Competitividad del calzado de cuero colombiano: Perspectiva de la ventaja comparativa revelada (1980-2001). Dimensión empresarial, 11(1), 77-91.

Gutiérrez, A. \& Rosales, M. (2010). La integración y las relaciones económicas entre Venezuela y Colombia: evolución reciente y perspectivas. Mundo Nuevo, 2, 267309.

Gutiérrez, A. \& Rosales, M. (2016). Modelo gravitacional del comercio entre Venezuela-Colombia ¿Es importante el libre comercio para aumentar el intercambio binacional? En: A. Gutiérrez (coor.), La integración económica entre Venezuela y Colombia: Evolución, balance y perspectivas (pp. 99-117). Mérida: Universidad de los Andes.

Gutiérrez, A. (2017). Venezuela y Colombia: divergencias en las estrategias de desarrollo, controversias e integración económica. Aldea Mundo, 22(44), 111-118.

Helpman, E. \& Krugman, P. R. (1985). Market structure and foreign trade: Increasing returns, imperfect competition, and the international economy. MIT press.

Hernández, J. (2009). Venezuela: Nota de Análisis Sectorial Agricultura y Desarrollo Rural. Organización de las Naciones Unidas para la Agricultura y la Alimentación (FAO), en: http://www.fao.org/docrep/012/ak170s/ak170s00.htm

Hoen, A. R. \& Oosterhaven, J. (2006). On the measurement of comparative advantage. Annals of Regional Science, 40(3), 677-691.

Krugman. P. \& Obstfeld, M. (2006). Economía internacional: teoría y política. Madrid: Pearson Education.

Lafay, G. (1987). Avantage comparatif et compétitivité. Economie prospective internationale, 29, 39-52.

Lampa, R. (2016). Crisis in Venezuela, or the Bolivarian dilemma: To revolutionize or to perish? A Kaleckian interpretation. Review of Radical Political Economics, 49(2), 198-218. 
Laursen, K. (2015). Revealed comparative advantage and the alternatives as measures of international specialization. Eurasian Business Review, 5(1), 99-115.

Linares, R. (2005). Zona de integración fronteriza (ZIF) y su dimensión territorial en la frontera Táchira (Venezuela)-Norte de Santander (Colombia). Aldea Mundo, 10(19), 45-54.

Malamud, C. (2006). La salida venezolana de la Comunidad Andina Naciones y sus repercusiones sobre la integración regional latinoamericana. Boletín Elcano, (86), 22.

Melcher, D. (1995). La industrialización de Venezuela. Revista Economía (10), 57-90.

Ministerio de Comercio, Industria y Turismo-MinCIT (2012). ABC del Acuerdo de Alcance Parcial suscrito entre Colombia y Venezuela. En: www.mincit.gov.co/publicaciones/4716/abc_del_acuerdo_de_alcance_parcial_suscrito_entre_colombia_ y_venezuela

Mojica, A. \& Paredes, J. (2004). La economía Colombo-venezolana y su impacto en la región de frontera. 1999-2003. Centro Regional de Estudios Económicos BucaramangaBanco de la República de Colombia, 1-25.

Moncayo, E. (2003). Geografía económica de la Comunidad Andina: Las regiones activas en el mercado comunitario. Bogotá.

Páez P.; Jiménez, W.; Danna-Buitrago J., (2018). La Competitividad de los Artículos de Calzado, Cuero y Marroquinería en Colombia: Revisión de la Literatura» Diálogos de Saberes, Universidad Libre. v.48. p.171-196.Palotas, L. (2008). Tres miradas al conflicto colombo-venezolano. Reflexión política, 10(19), 60-72.

Perdomo, J. \& Malaver, F. (2002). Informe final Proyecto equipo negociador del ALCA en su componente de competitividad. Universidad Nacional de Colombia.

Preusse, H. (2007). Consecuencias de la salida de Venezuela de la CAN y su entrada al MERCOSUR. En Z. Drna (Coor.), Mercosur y Unión Europea (pp. 181-198). Argentina: Lerner.

Porter, M. (1990). The Competitive Advantage of Nations. Free Press, New York.

Ricardo, D. (1817). On the Principles of Political Economy and Taxation, London: John Murray.

Romero, C. (2010). La política exterior de la Venezuela bolivariana. Rio de Janeiro: Plataforma democrática. 
Romero, A., Monroy, R. \& Ramírez, R. (2017). Estrategias para mejorar la productividad y competitividad de las empresas de calzado de Cúcuta. Espacios, 38(39), 1-10.

Ruiz, D. \& Rosales, M. (2009). Comercio bilateral Venezuela-Colombia 1999-2008. Aldea Mundo. Revista sobre fronteras e integración, 14(27), 17-25.

Sánchez, F. (2011). La frontera Táchira (Venezuela)-Norte de Santander (Colombia) en las relaciones bi-nacionales y en la integración regional. Si somos americanos, Revista de estudios transfronterizos, 11(1), 63-84.

Sannassee, V., Seetanah, B. \& Jugessur, J. (2014). Export-led Growth Hypothesis: A Meta-Analysis. Journal of Developing Areas, 48(1), 361-385.

Stellian, R. \& Danna-Buitrago, J. P. (2017a). Competitividad de los productos agropecuarios colombianos en el marco del tratado de libre comercio con los Estados Unidos: análisis de las ventajas comparativas. Revista CEPAL, 122, 139-163.

Stellian, R. \& Danna-Buitrago, J. P. (2017b). Protocolo de medición de las ventajas comparativas de los artículos manufacturados colombianos frente a Estados Unidos. En J. P. Danna-Buitrago. (Ed.), Análisis retrospectivo de la integración internacional del sector manufacturero colombiano en el marco del Tratado de Libre Comercio con Estados Unidos (pp. 55-67). Bogotá: Los Libertadores.

Urrutia, M., Posada, E., Pontón, A. \& Martínez, O. (2000). Comercio exterior y actividad económica de Colombia en el siglo XX: Exportaciones totales y tradicionales (nro. 163). Banco de la República de Colombia.

Vera, L. (2018). Cómo explicar la catástrofe económica venezolana. Nueva Sociedad, (274), 83-96.

Villegas, C. \& Zapata, D. (2007). Competitividad sectorial internacional caso: Sector del cuero y del calzado. Entramado, 3(1), 24-49.

Yu, R., Cai, J. \& Leung P. (2009). The normalized revealed comparative advantage index. Annals of Regional Science, 43(1), 267-282. 
